
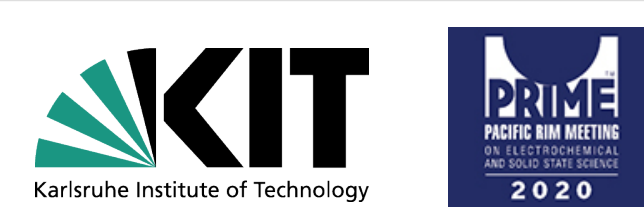

\title{
Late A - 3730: Progress in thermal management and safety of cells and packs by testing in battery calorimeters
}

C. Ziebert (carlos.ziebert@kit.edu), N. Uhlmann, I. Mohsin, M. Rohde, H. J. Seifert Institute for Applied Materials - Applied Materials Physics (IAM-AWP)

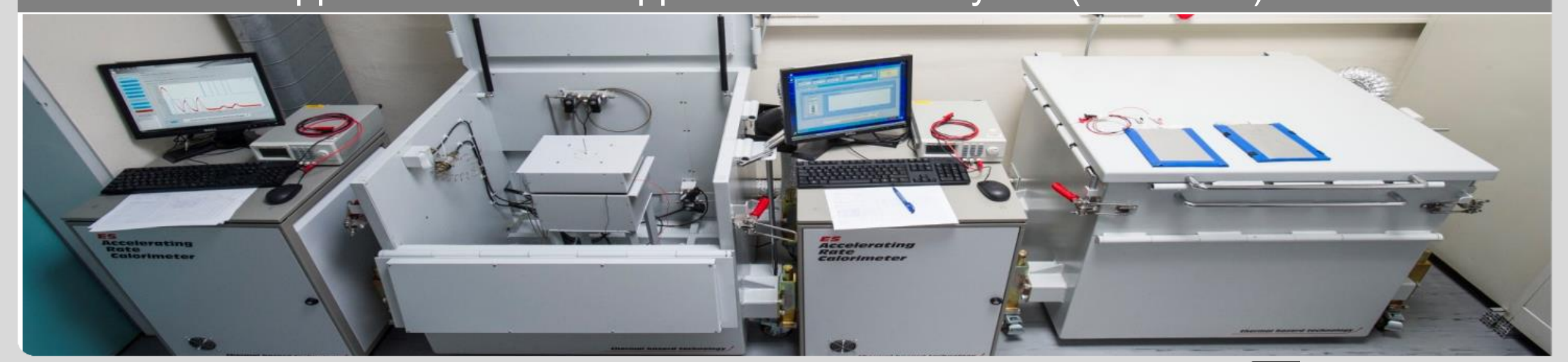




\section{Motivation}

\section{Causes and effects of thermal runaway}
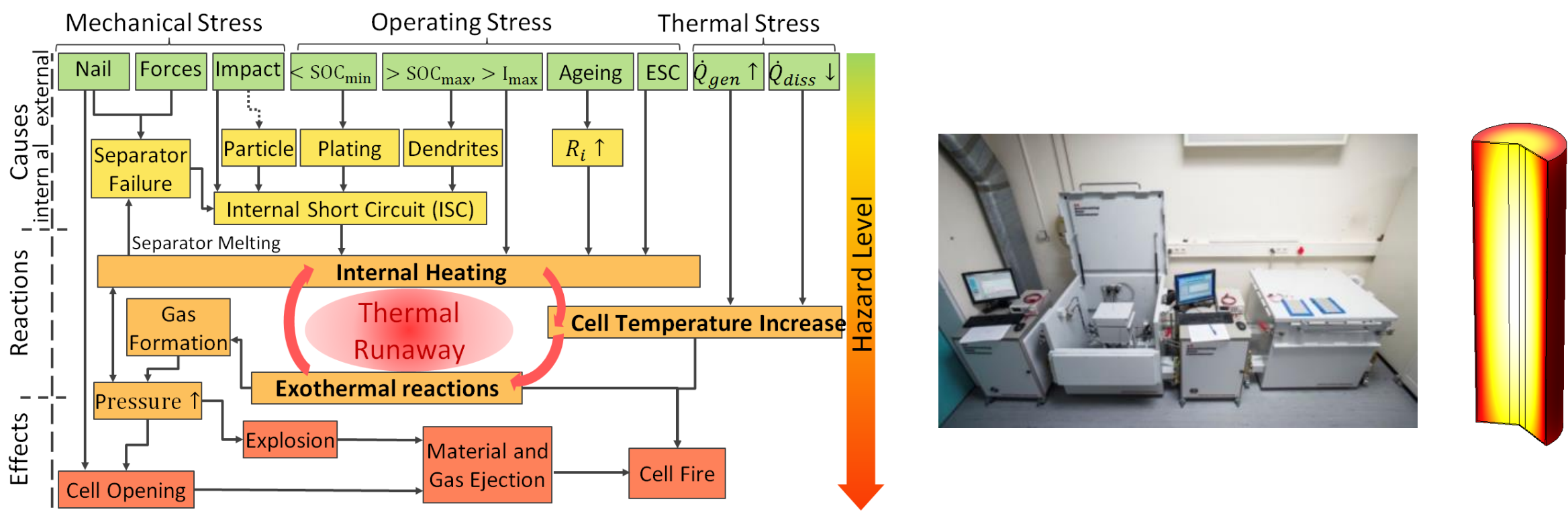

Aim: Improvement of battery management, thermal management and safety systems by determination of quantitative data using battery calorimetry in combination with modelling and simulation 


\section{Overview}

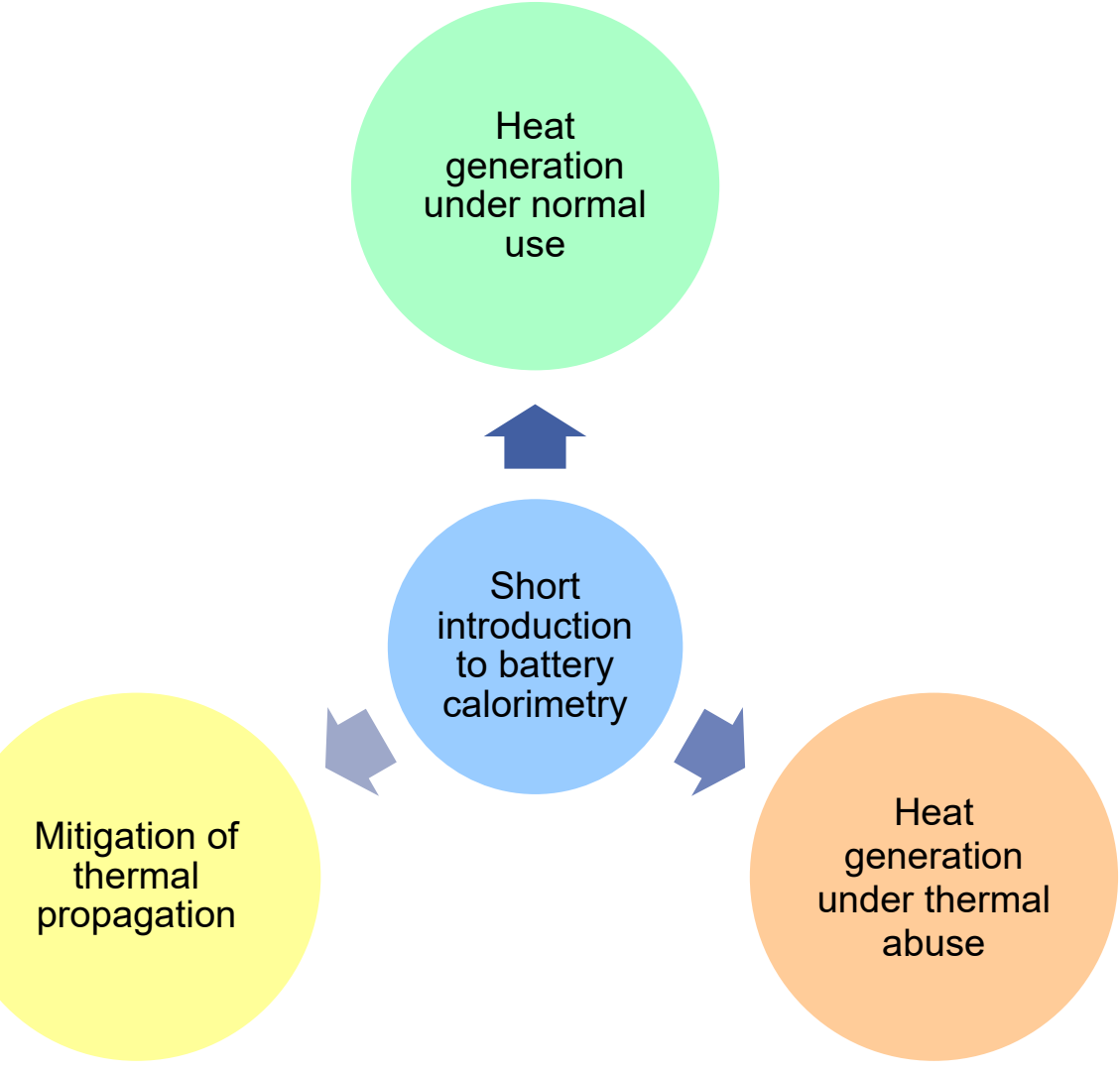




\section{Overview}

Heat

generation

under normal

use

Mitigation of

thermal

propagation

Heat

generation

under thermal

abuse 


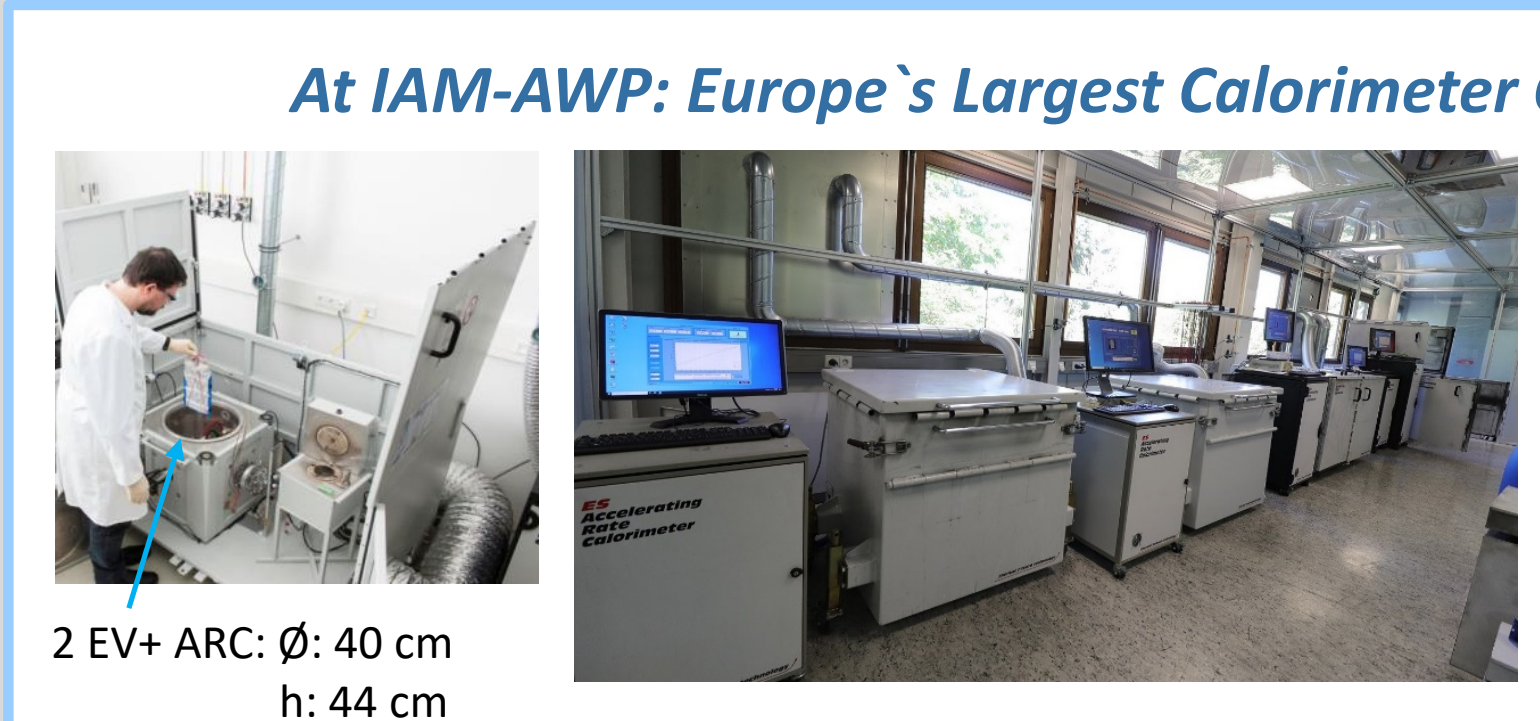

\section{Center}

$\mathbf{S}$ II

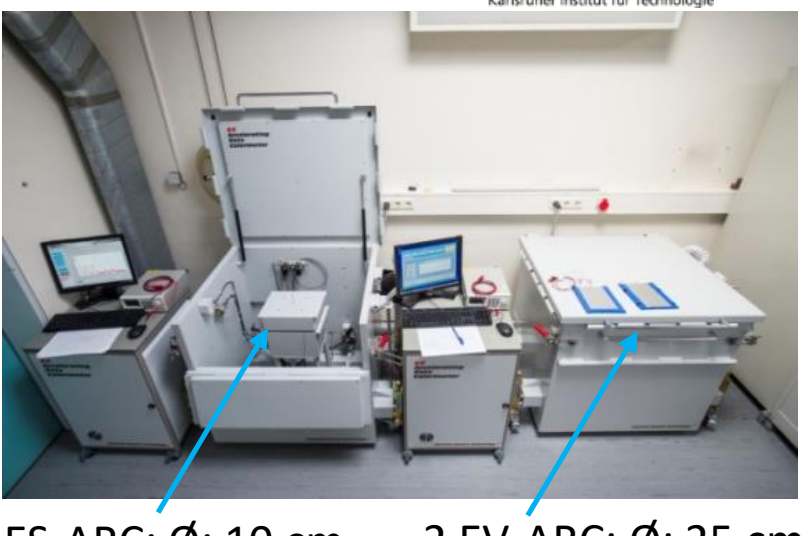

2 ES-ARC: $\varnothing: 10 \mathrm{~cm}$

2 EV-ARC: $\varnothing: 25 \mathrm{~cm}$

h: $10 \mathrm{~cm}$

h: $50 \mathrm{~cm}$

Equipment: 6 ARC's (THT); 2 Tian-Calvet calorimeters (C80, MS80: Setaram); 4 DSC (Netzsch); IR camera (FLIR); 13 Temperature chambers; 11 Cyclers; EIS (Ref3000, Gamry)
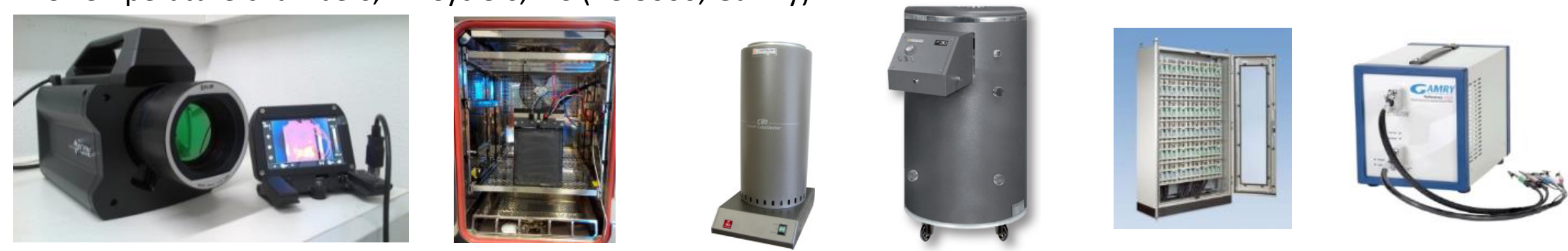


\section{Short introduction to battery calorimetry}

\section{Cell types that can be investigated in battery calorimeters}

Coin cells

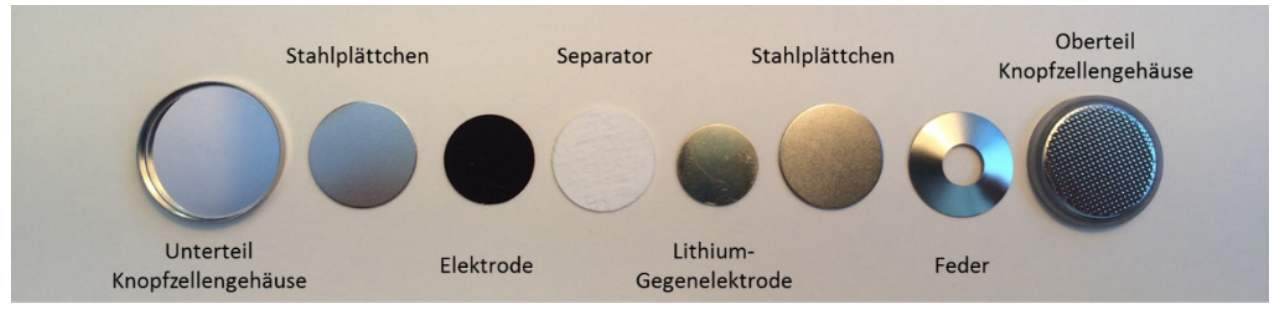

Cylindrical cells, e.g. 18650,21700

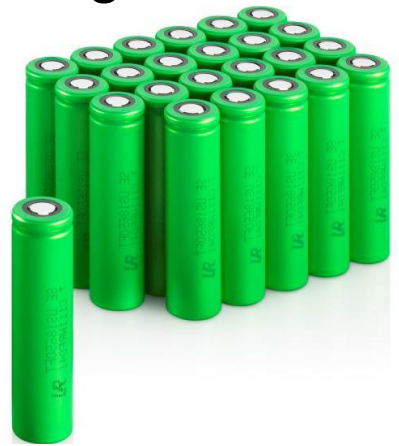

Prismatic cells

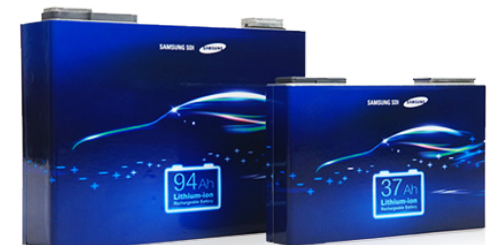

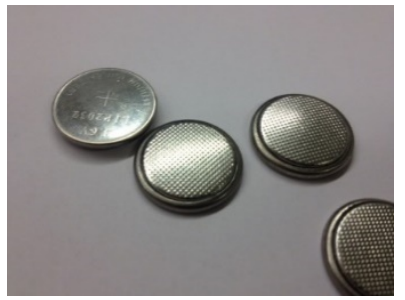

Pouch cells

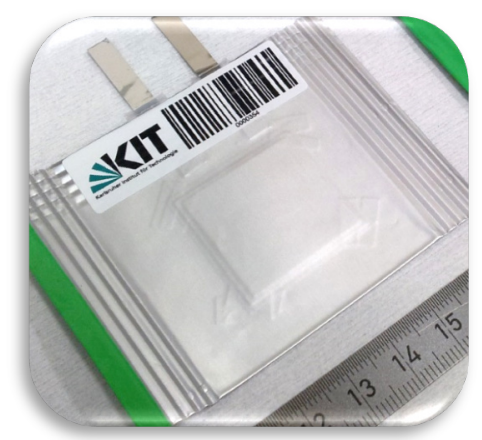




\section{Possible conditions in an Accelerating Rate Calorimeter (ARC)}

An ARC provides isoperibolic and adiabatic conditions

Under isoperibolic conditions the environmental temperature is kept constant.
Under adiabatic conditions the heaters follow

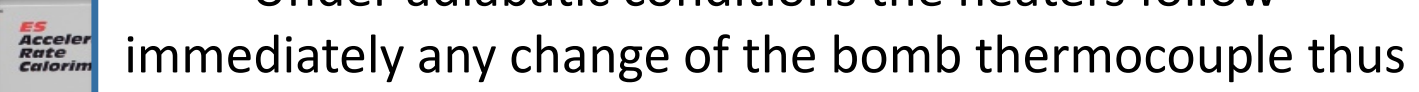
preventing that the cell can transfer heat to the walls.

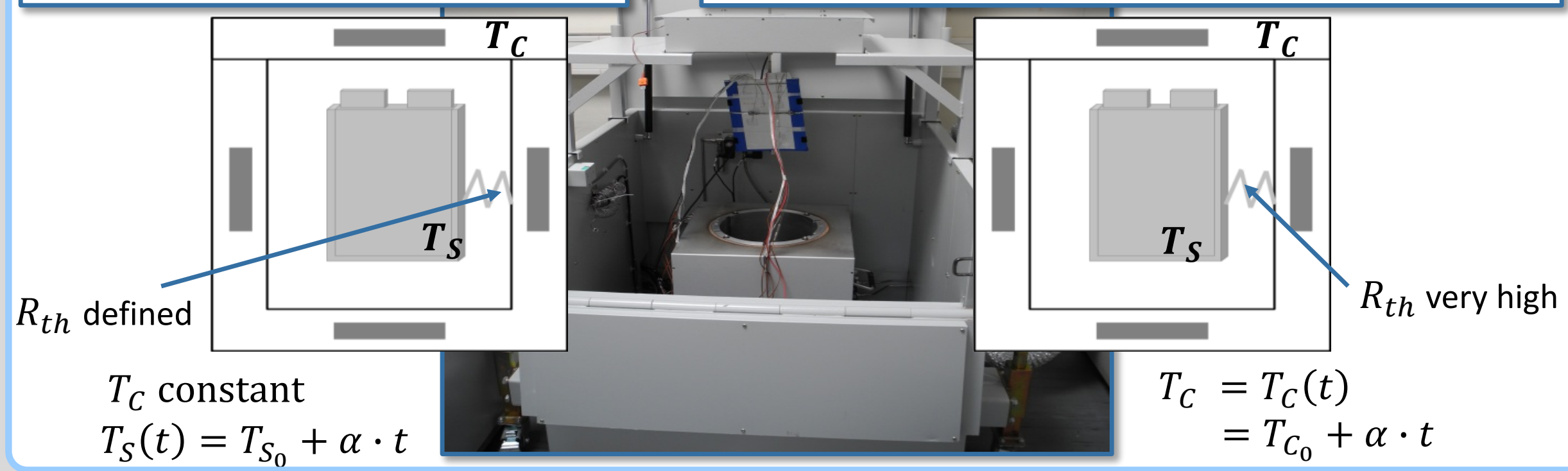




\section{Overview}

Heat

generation

under normal

use

Mitigation of

thermal

propagation

Heat

generation

under thermal

abuse 


\section{Heat generation under normal use}

\section{Measurements in the MS80 Tian-Calvet Calorimeter on Na-ion coin cell}

Cathode: $\mathrm{Na}_{0.53} \mathrm{MnO}_{2}$

Anode: Hard carbon

Electrolyte: $1 \mathrm{M} \mathrm{NaClO}_{4}$ [EC:DMC:EMC (vol. 1:1:1) 2\% FEC]

\section{Charge parameter}

(CCCV) Profile at $25^{\circ} \mathrm{C}, \mathrm{CV}$-Step at $4.0 \mathrm{~V}(\mathrm{I}<\mathrm{C} / 20$ or $\mathrm{t}>60 \mathrm{~min})$

\section{Discharge parameter}

(CCCV) Profile at $25^{\circ} \mathrm{C}, \mathrm{CV}$-Step at $2.0 \mathrm{~V}(\mathrm{I}<\mathrm{C} / 20$ or $\mathrm{t}>60 \mathrm{~min})$

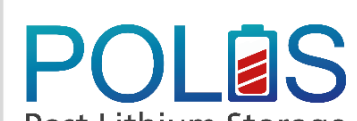

Post Lithium Storage Cluster of Excellence

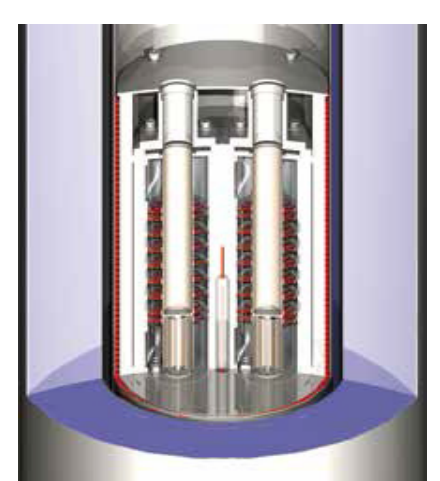

Vessel $\varnothing: 32 \mathrm{~mm}$

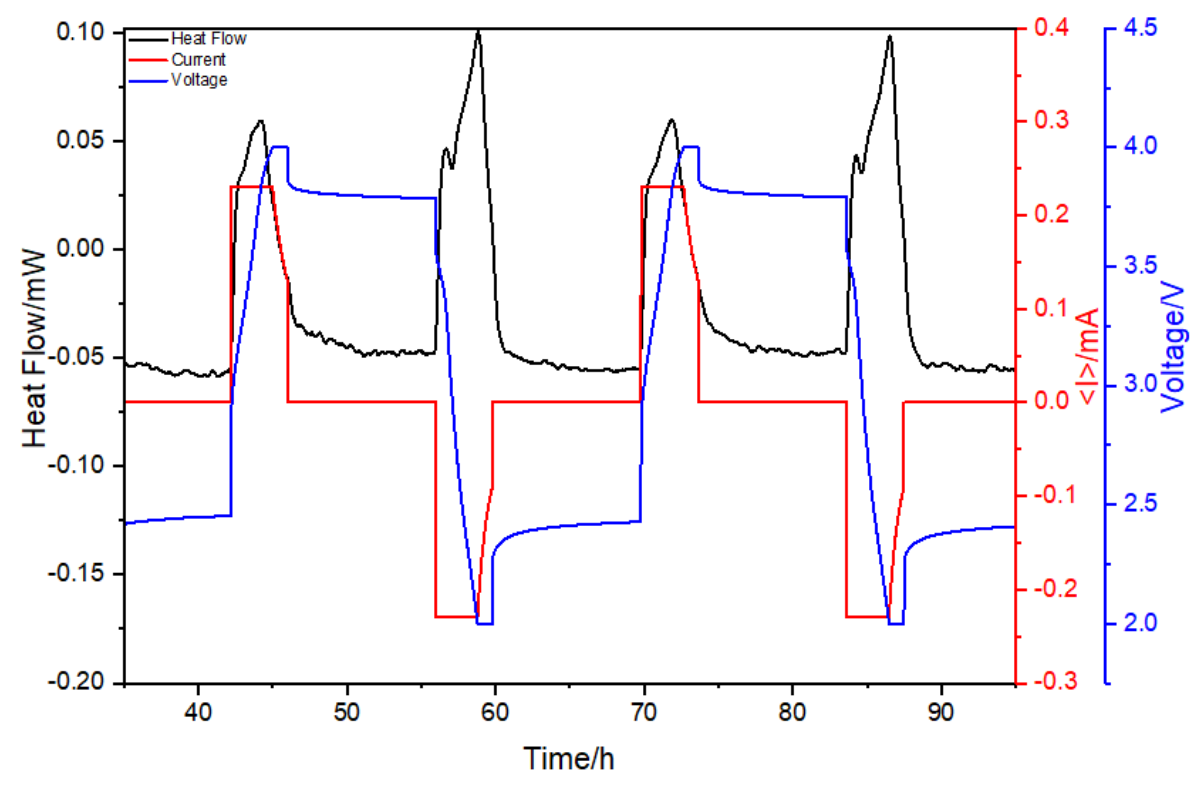

\begin{tabular}{|c|c|c|c|}
\hline $\begin{array}{c}\text { Current Flow } \\
(1.15 \mathrm{mAh})\end{array}$ & $\begin{array}{c}\text { Capacity } \\
\mathrm{mAh}\end{array}$ & $\begin{array}{c}\text { Heat generation } \\
\text { charge }(\mathrm{J})\end{array}$ & $\begin{array}{c}\text { Heat generation } \\
\text { discharge }(\mathrm{J})\end{array}$ \\
\hline $0.2 \mathrm{C}$ & $0.82 \pm 0.04$ & $1.31 \pm 0.03$ & $1.49 \pm 0.01$ \\
\hline
\end{tabular}


Worst Case Conditions

$\rightarrow$ Cell in a pack surrounded by other cells

Discharge parameter:

- method: constant current (CC)

- $U_{\text {min }}=3.0 \mathrm{~V}$

- $\mathrm{I}=5 \mathrm{~A} \rightarrow \mathrm{C} / 8$-rate

Charge parameter:

- method: constant current, constant voltage (CCCV)

- $\quad U_{\max }=4.1 \mathrm{~V}$

- $\quad \mathrm{I}=5 \mathrm{~A} \rightarrow \mathrm{C} / 8$-rate

- $\mathrm{I}_{\min }=0.5 \mathrm{~A}$

$\rightarrow$ after each electrochemical cycle the cell temperature increases further

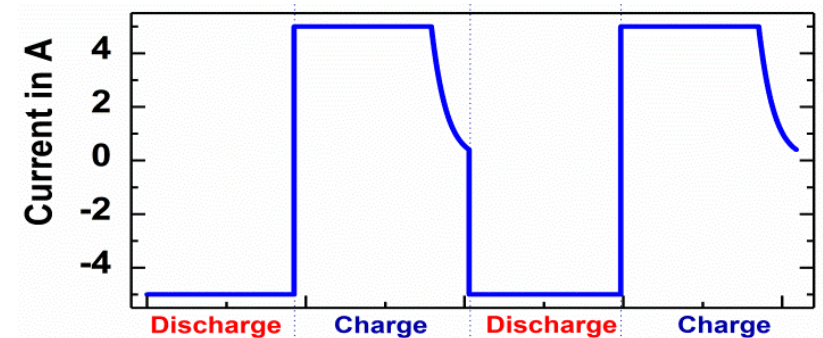

40 Ah pouch cell

NMC111/graphite
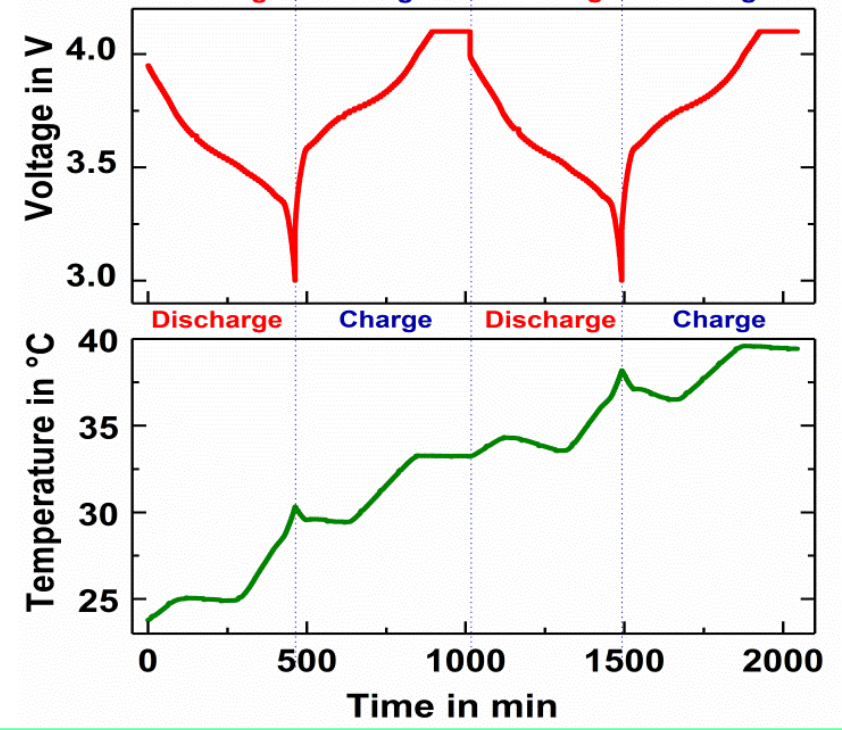

$T_{s t}=23^{\circ} \mathrm{C}(\mathrm{RT})$ 


\begin{tabular}{ll|}
\hline Discharge parameter: \\
$-\quad$ method: constant current (CC) \\
$-\quad U_{\min }=3.0 \mathrm{~V}$ \\
$-\quad \mathrm{I}=5 \mathrm{~A} \rightarrow \mathrm{C} / 8$-rate \\
Charge parameter: \\
$-\quad$ method: constant current, \\
$\quad$ constant voltage (CCCV) \\
$-\quad U_{\max }=4.1 \mathrm{~V}$ \\
$-\quad \mathrm{I}=5 \mathrm{~A} \rightarrow \mathrm{C} / 8$-rate \\
$-\quad \mathrm{I}_{\min }=0.5 \mathrm{~A}$
\end{tabular}

$\rightarrow$ after one electrochemical cycle the cell temperature reaches its initial value again
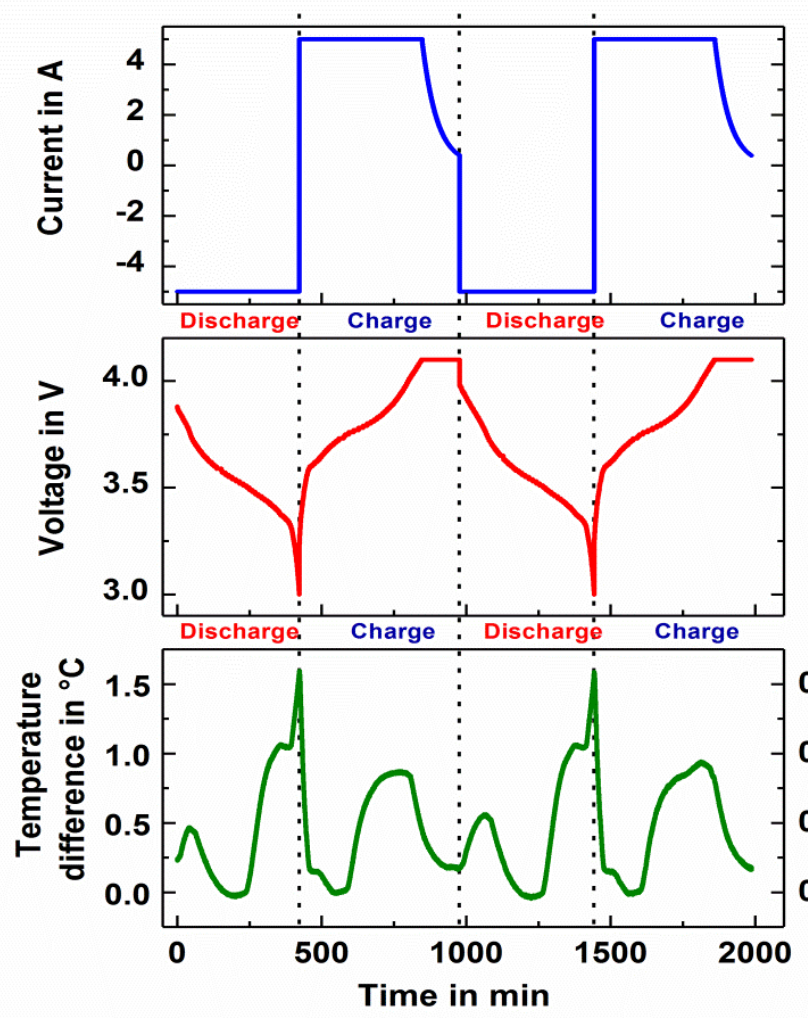

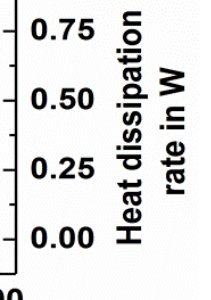

$\left(\frac{\delta E}{\delta T}\right)<0$

temperature coefficient negative! 


\section{Comparison of the values for the generated heat}

determined by three different methods

1) Adiabatic Measurement

$$
\dot{Q}_{g}=m c_{p} \frac{d T}{d t}
$$

2) Isoperibolic Measurement

$$
\dot{Q}_{g}=m c_{p} \frac{d T}{d t}+A h \cdot\left(T_{S}-T_{C}\right)
$$

3) Measurement of irreversible and reversible heat

$$
\dot{Q}_{g}=-I\left(E_{0}-E\right)-I T \frac{d E_{0}}{d T}
$$

$E_{0}$ : Open circuit voltage (OCV), E: cell potential

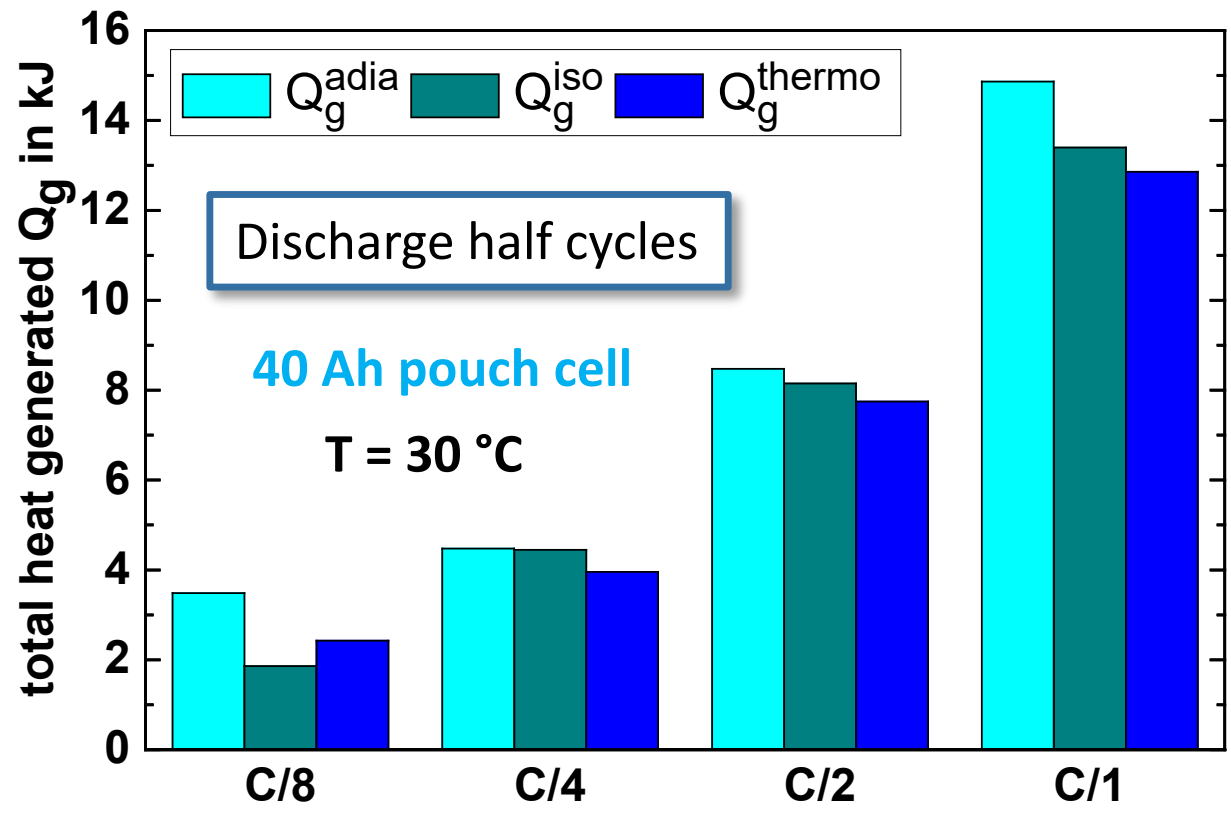

discharge rate

Conclusion: good agreement between the values determined by the different methods E. Schuster, C. Ziebert, A. Melcher, M. Rohde, H.J. Seifert, J. Power Sources 268 (2015) 580-589 


\section{Overview}

Heat

generation

under normal

use

Mitigation of

thermal

propagation

Heat

generation

under thermal

abuse 


\section{Heat generation under thermal abuse}

Heat-Wait-Seek(HWS) Method in ARC
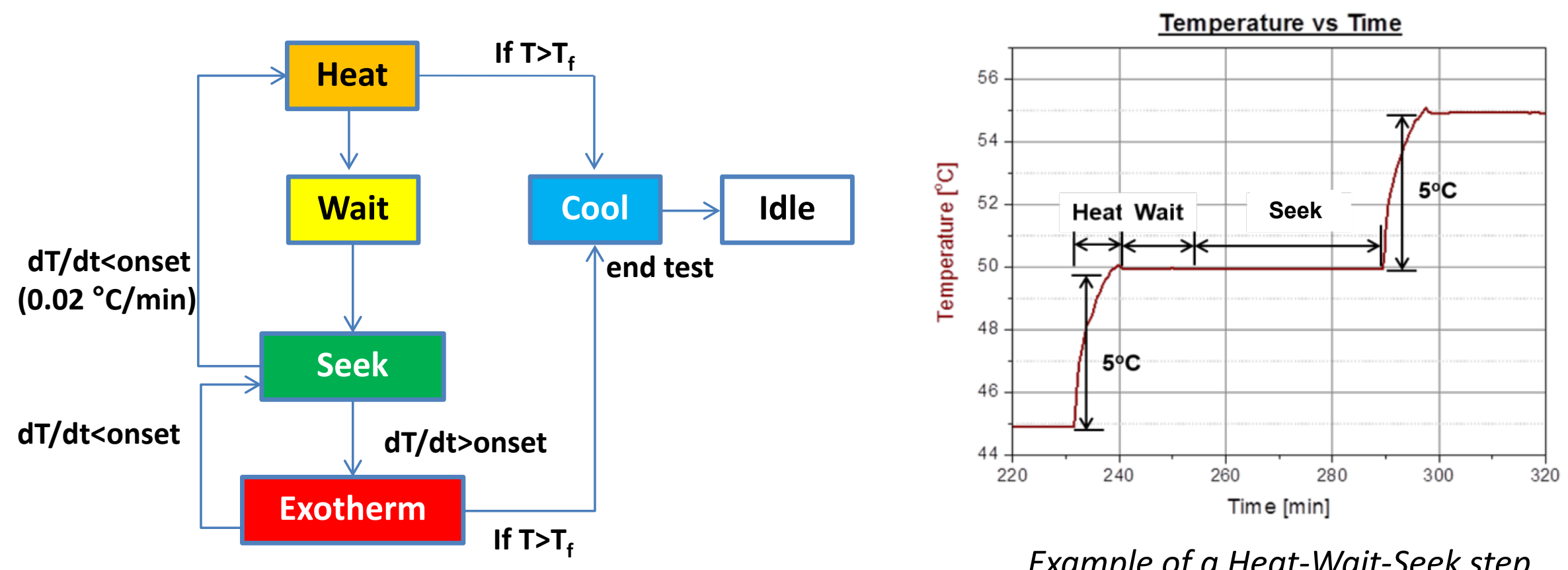

Example of a Heat-Wait-Seek step

C. Ziebert, A. Melcher, B. Lei, W.J. Zhao, M. Rohde, H.J. Seifert, Electrochemical-thermal characterization and thermal modeling for batteries, in: L.M. Rodriguez, N. Omar, Eds., EMERGING NANOTECHNOLOGIES IN RECHARGABLE ENERGY STORAGE SYSTEMS, EIsevier Inc. 2017 , ISBN 978032342977. 


\section{Thermal Runaway: 18650 Li-ion cells with different cathode materials}
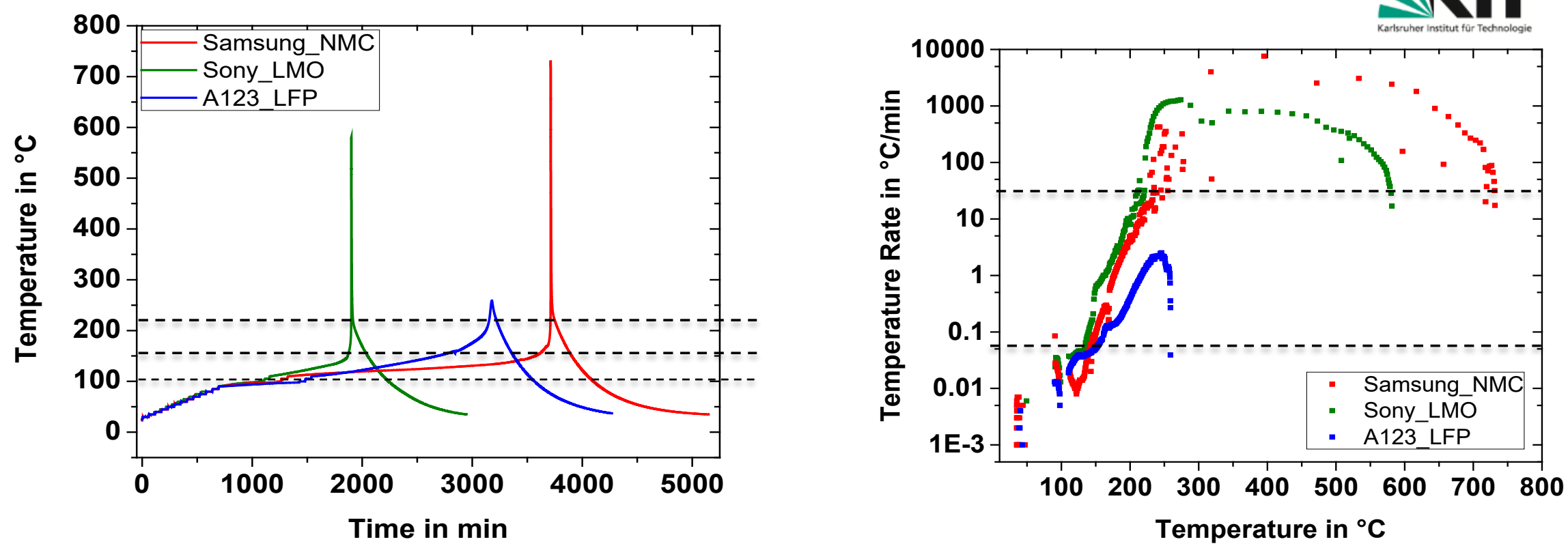

- $80<\mathrm{T}<130^{\circ} \mathrm{C}$ : low rate reaction, $0.02-0.05^{\circ} \mathrm{C} / \mathrm{min}$ : exothermic decomposition of the SEI

- $130<\mathrm{T}<200^{\circ} \mathrm{C}$ : medium rate reaction, $0.05-25^{\circ} \mathrm{C} / \mathrm{min}$ : solvent reaction, exothermic reaction between embedded Li ions and electrolyte $\Rightarrow>$ reduction of electrolyte at negative electrode

- T $>200^{\circ} \mathrm{C}$ : high rate reaction, higher than $25^{\circ} \mathrm{C} / \mathrm{min}$ : Exothermic reaction between active positive material and electrolyte at positive electrode $=>$ rapid generation of oxygen 


\section{Development of internal pressure measurement methods}

for 18650 cells
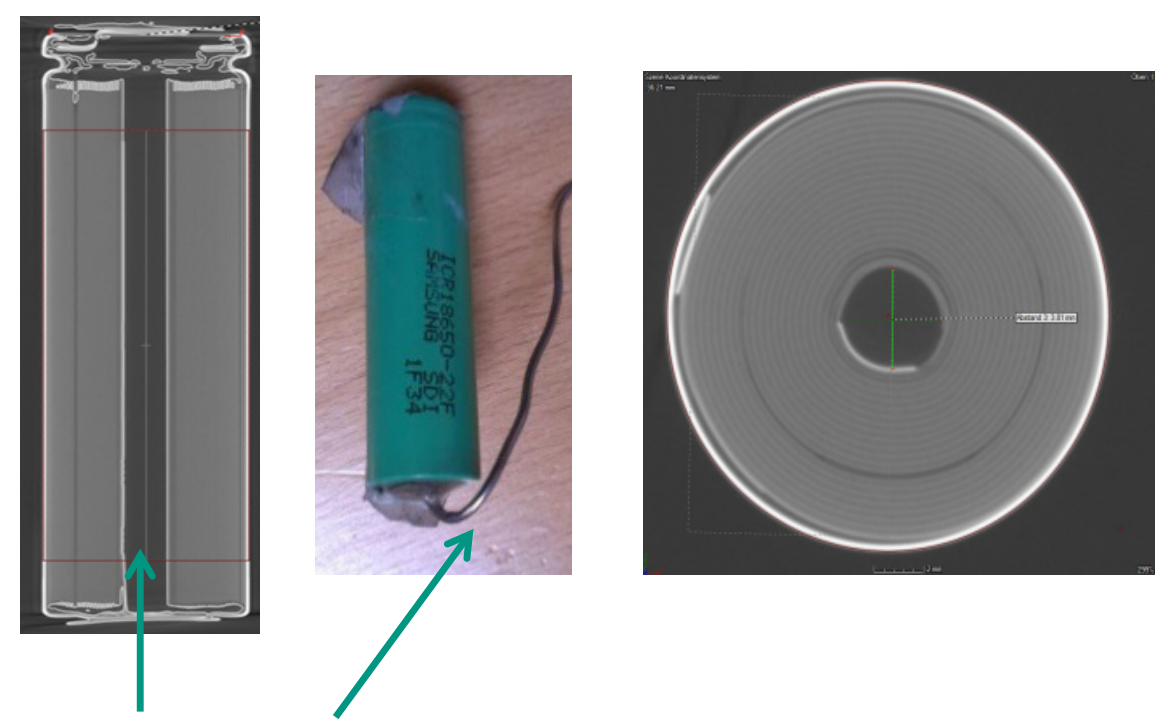

Pressure line $(\varnothing 1.5 \mathrm{~mm})$
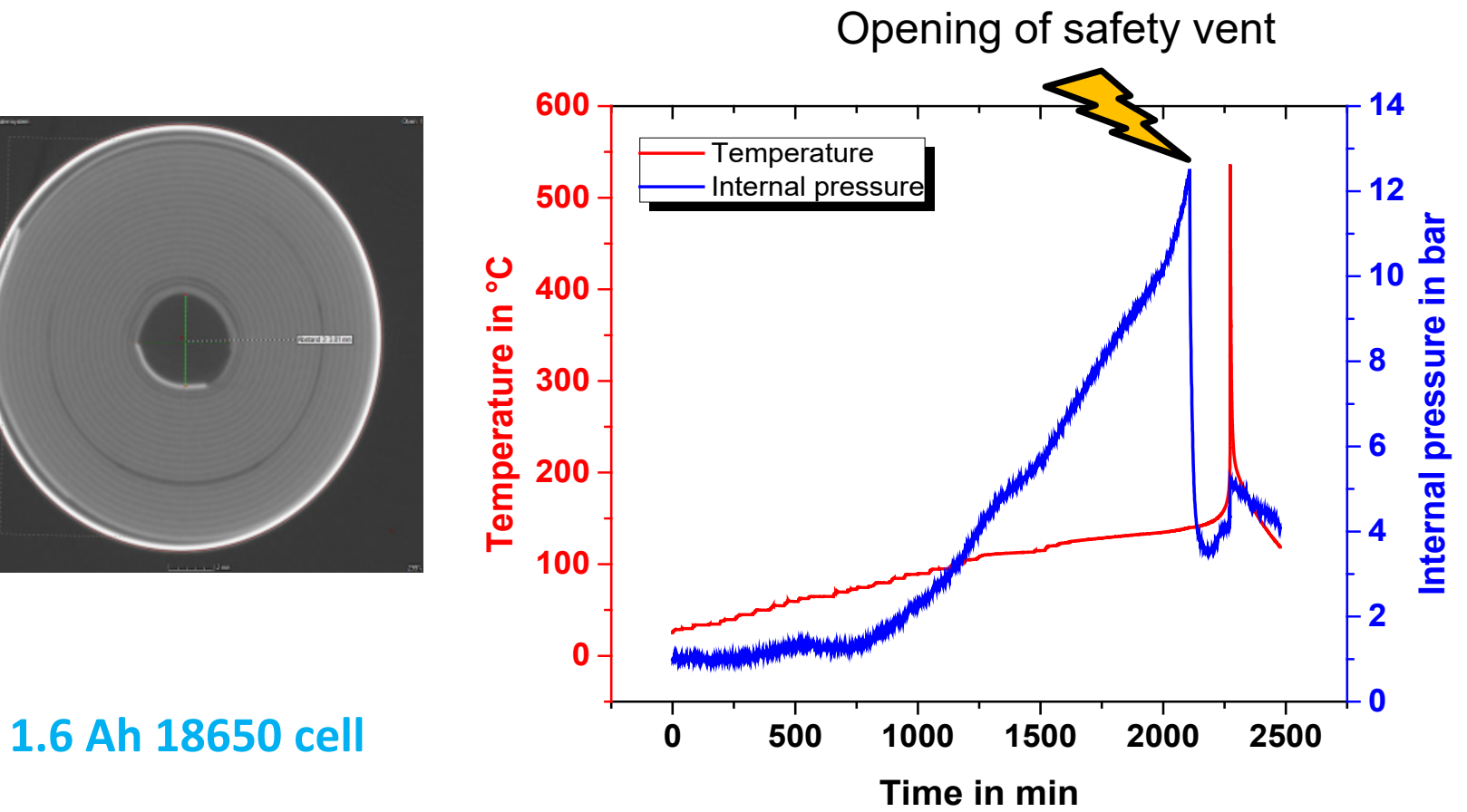

Internal pressure could be used in BMS for early prediction of processes leading to thermal runaway

B. Lei, W. Zhao, C. Ziebert, A. Melcher, M. Rohde, H.J. Seifert, Batteries 2017, 3, 14, doi:10.3390/batteries3020014. 


\section{Overview}

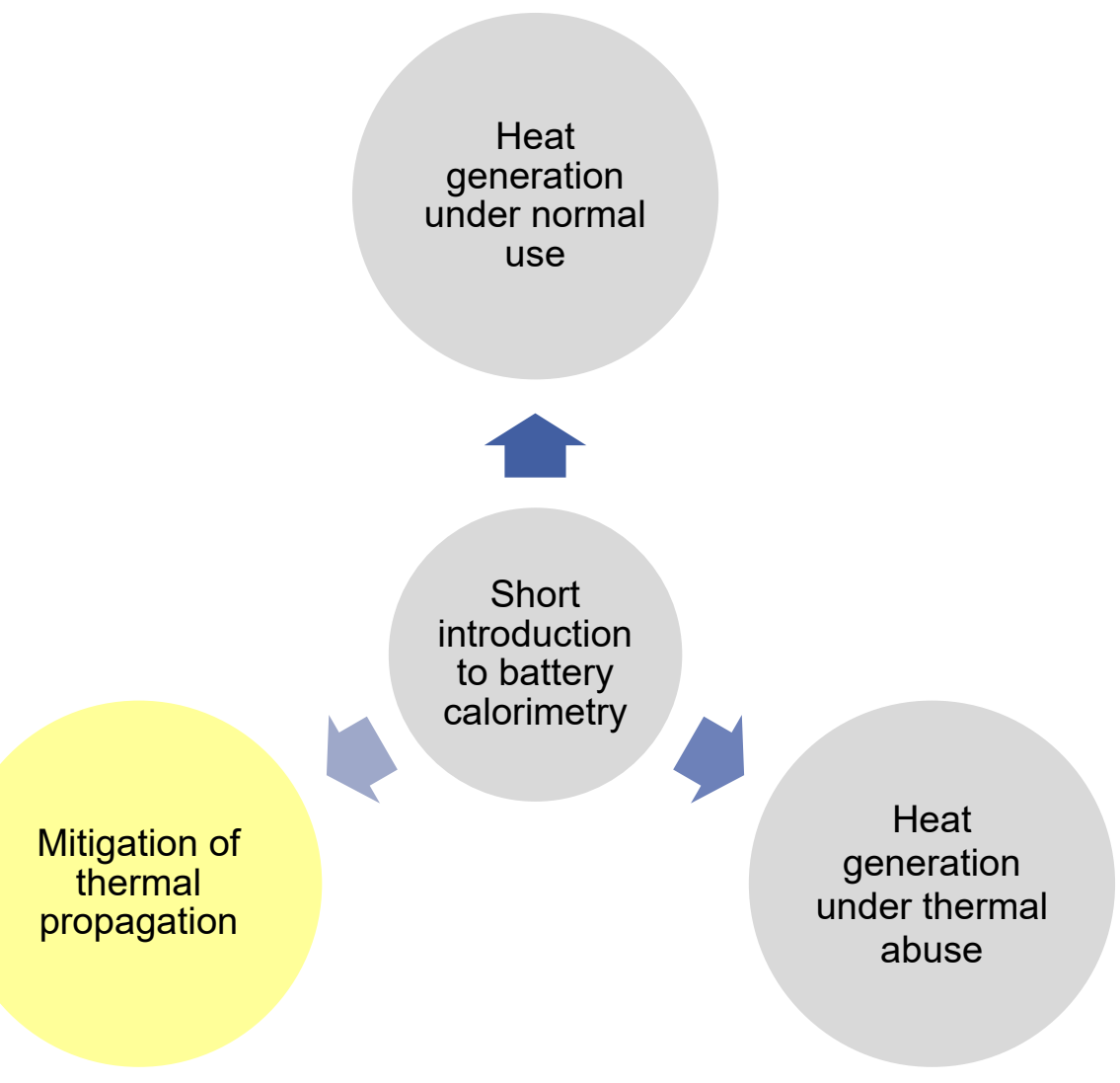




\section{Material qualification for passive propagation prevention}
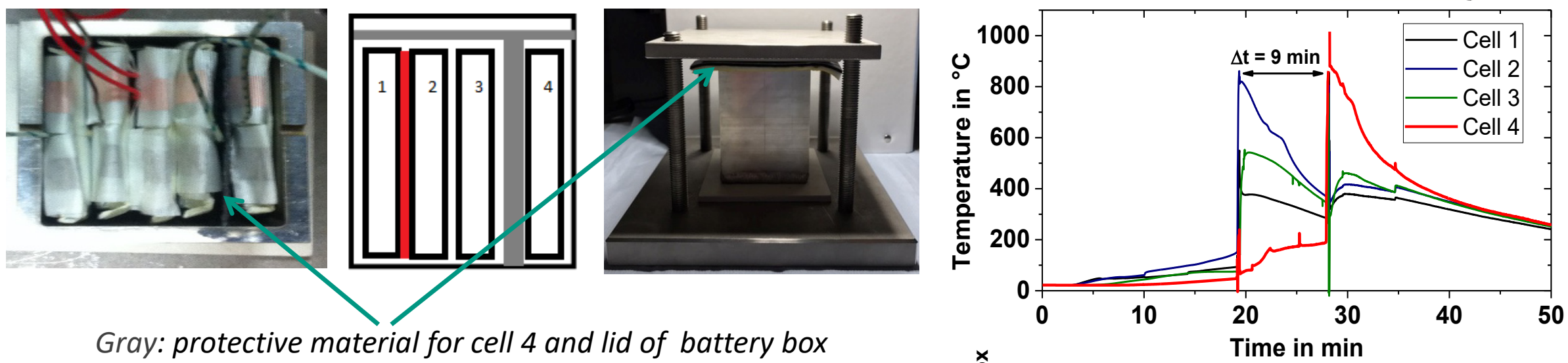

Gray: protective material for cell 4 and lid of battery box

Red: heater mat for thermal runaway initiation

\section{4 x 4.5 Ah Ah pouch cell \\ NMC111/graphite}

\section{Optimized Multilayer: HKO-Defensor ML 14}

- Extended time for propagation: $9 \mathrm{~min}$

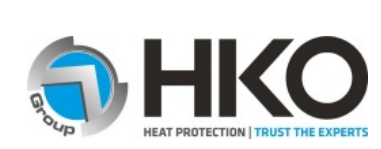

- Improved heat protection: temperature on top of battery box $<80^{\circ} \mathrm{C}$ during thermal runaway

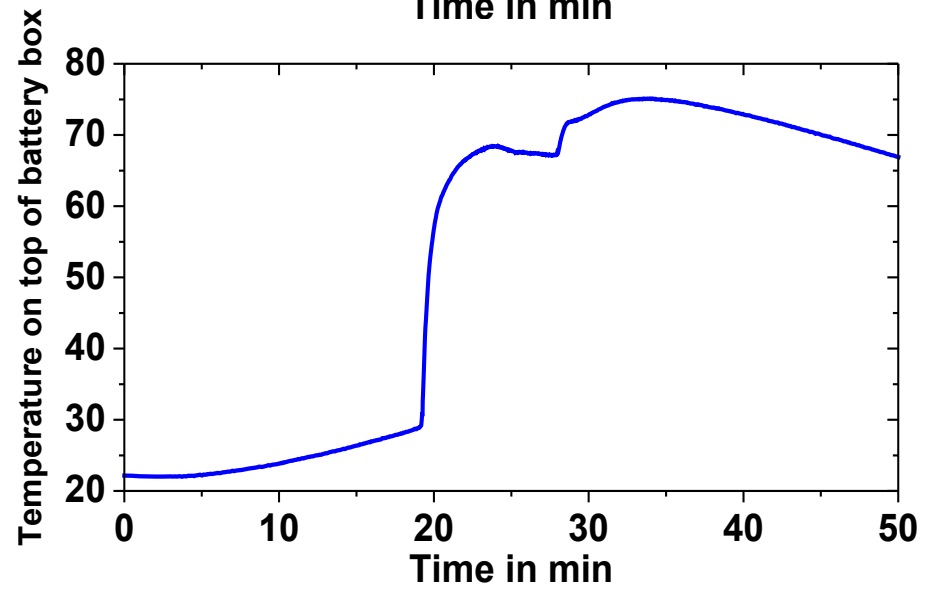




\section{Summary: Possible measurements with a battery calorimeter}

Normal conditions of use

- Isoperibolic or adiabatic measurement

Measurement of temperature curve and temperature distribution during cycling (full cycles,

For each: or application-specific load profiles), ageing studies

Determination of the generated heat, Separation of heat in reversible and irreversible parts

\section{Abuse conditions}

- Thermal abuse: Heat-wait-seek test, ramp heating test, thermal propagation test

- Mechanical abuse: Nail penetration test

- Electric abuse: Overcharge, external short circuit

$>$ Temperature measurement

For each: $>$ External or internal pressure measurement

$>$ Gas collection, Post Mortem Analysis, Ageing studies

\section{Contact: \\ Phone: $\quad++49 / 721608-22919$ \\ E-Mail: $\quad$ Carlos.Ziebert@kit.edu}




\section{Thank you for your kind attention

This work has been partially funded by the Federal Ministry for Education and Research (BMBF) within the framework "IKT 2020 Research for Innovations" under the grant 16N12515 and was supervised by the Project Management Agency VDI|VDE|IT.

This work contributes to the research performed at CELEST (Center for Electrochemical Energy Storage Ulm-Karlsruhe) and was funded by the German Research Foundation (DFG) under Project ID 390874152 (POLiS Cluster of Excellence).

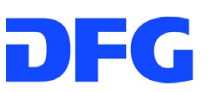

Forschungsgemeinschaf German Research Foundation

Additional funding by the Helmholtz Association is gratefully acknowledged.

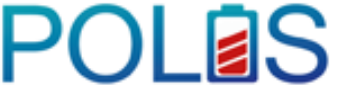

Post Lithium Storage Cluster of Excellence

HELMHOLTZ

ASSOCIATION 

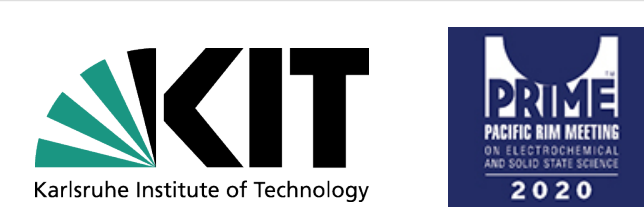

\section{Late A - 3730: Progress in thermal management and safety of cells and packs by testing in battery calorimeters}

C. Ziebert (carlos.ziebert@kit.edu), N. Uhlmann, I. Mohsin, M. Rohde, H. J. Seifert Institute for Applied Materials - Applied Materials Physics (IAM-AWP)

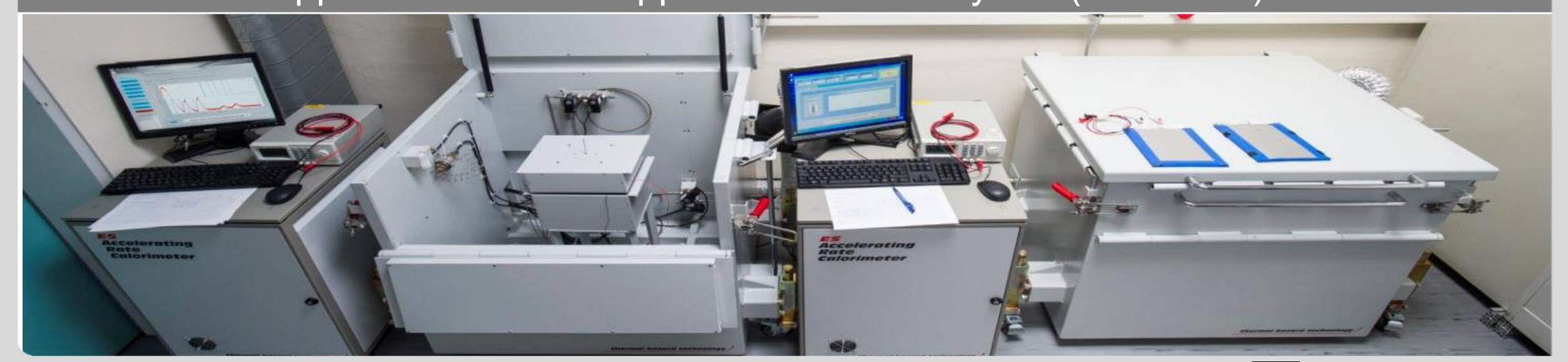

